\title{
Perception Of Oral Hygiene Practices And Dental Treatment Permissible During Fasting Amongst Dental Patients: A Cross-Sectional Study In Pakistan
}

Khaloud Tariq ( $\sim$ khaloud.tariq@ucd.uol.edu.pk)

University of Lahore https://orcid.org/0000-0002-1325-593X

Arooj UI Hassan

The University of Lahore University College of Medicine and Dentistry

Muhammad Hassan

The University of Lahore University College of Medicine and Dentistry

Shahab Ud Din

Shaheed Zulfiqar Ali Bhutto Medical University

Zunaira Arooj

The University of Lahore

Moghees A. Baig

The University of Lahore University College of Medicine and Dentistry

Research article

Keywords: Fasting, Oral Hygiene, Dental treatment

Posted Date: January 29th, 2020

DOI: https://doi.org/10.21203/rs.2.22096/v1

License: (c) (1) This work is licensed under a Creative Commons Attribution 4.0 International License.

Read Full License 


\section{Abstract}

Background: Fasting places prohibitions on eating and drinking for a certain period of time. Although many dental treatments have been said to be safe and can be performed while fasting, other may result in breaking of fast. The study aims to evaluate the perception of dental patients of Lahore, Pakistan regarding effect of such treatments and hygiene measures during fasting. Methods : It was a crosssectional survey carried out in 2 dental teaching hospitals of Lahore, Pakistan from SeptemberOctober 2018. Patients were asked to complete a self-administered structured questionnaire developed to assess the knowledge regarding dental treatments, and hygiene practices while fasting. The questionnaire consisted of 7 items regarding the influence of dental treatments such as filling, scaling, and extraction etc. on fasting. Questions were also added regarding maintenance of oral hygiene such as brushing and its effect on fast. Frequencies and percentages are used to display results. Chi-squared test was used for statistical analysis to estimate difference between gender, and educational status with perception of procedures breaking fast. Results : Out of 415 responses, 374 were included for analysis. About $76.2 \%$ respondents believed that undergoing extraction broke fast. Scaling was thought to nullify the fast by $45.5 \%$ of respondents. $52.9 \%$ perceived root canal treatment to break fast along with $67.6 \%$, who believed anaesthesia administration broke fast. As far as oral hygiene was concerned, brushing was reported to break fast by $57.5 \%$ with the use of mouthwash invalidating fast by $63.4 \%$. Conclusion : The respondents were generally aware of some procedures not breaking fast however, most thought that administration of anaesthesia, undergoing root canal treatment, and extraction invalidated fast. Also, hygiene maintenance measures such as brushing and use of mouthwash during was also thought to break fast. It is imperative that the healthcare providers should be familiar with the effect of dental treatments and oral hygiene measures on fasting state that can influence the treatment plan of the patients.

\section{Background}

Fasting is an obligatory practice in Islam during the month of Ramadan. According to Islamic calendar (Hijra), each year Ramadan comes about 10 days earlier thus, occurring at various times in different seasons in a 33-year cycle. ${ }^{1}$ One of the fundamentals of fasting is totally refraining oneself from consuming any food or drink during the fasting hours. Although traditions may vary according to geographical location, customs are generally influenced by religion specially in regions like middle east and southeast Asia. This phenomenon is reflected in people having same thought process, as they are led by the same ideology. As per Islamic teachings, people who are unwell, elderly and traveling are not covered under the same umbrella of compulsory fasting. ${ }^{2}$ It also includes women who are menstruating, pregnant, new mothers and lactating. Therefore, such individuals have the right to be excused from fasting which can be performed later.,

Fasting applies some restrictions on the Muslims who are observing it in the terms of what could potentially break a fast. These limitations include ingesting any solid or intake of minute quantity of water; which can nullify the fast. This places patients and healthcare professionals in a predicament 
where, patients will have to ascertain the type of medication they can take before or after fasting along with any diagnostic test or medical/dental procedure. Moreover, practitioners will also have to consider modifications in treatment plans and medication timing so that it doesn't hinders the obligation of performing fast. As far as dental procedures are concerned, it can be assumed that general population is relatively unfamiliar with ramifications of different treatments on fasting. This can be inferred from the fact that most people inquire about the effect of oral hygiene practices such as brushing and use of mouthwash on fasting. ${ }^{5}$ Also, the ratio of attending dental appointments have been documented to decline during Ramadan, which not only delays the treatment but may lead to poor oral health outcomes as well. ${ }^{6,7}$ It can only be expected that they will have little knowledge about permissible dental treatments during Ramadan.

Thus, information about the patients' cultural and religious rituals is of great importance for healthcare professionals, as this knowledge will result in gratification of patients regarding dental healthcare delivery. ${ }^{3}$

Therefore, the aim of this study was to assess the level of awareness among the patients regarding the types of treatments and oral hygiene practices that are permissible during fasting.

\section{Objectives}

- To estimate perception of respondents with regards to dental treatments and hygiene practices that can break fast.

- To identify any difference in perception of dental treatments and hygiene practices amongst genders.

- To evaluate any difference in perception among participants of different educational status.

\section{Methods}

The study was a cross-sectional survey carried out in OPD's of two dental teaching hospitals of Lahore, Pakistan affiliated with De 'Montmorency College of Dentistry and University College of Dentistry, University of Lahore from September-October 2018. Sample size was calculated using online Raosoft sample size calculator, with population size $(43,200)$ projected as annual visitors in OPD of both dental teaching hospitals. Keeping margin of error $5 \%$ and confidence interval $95 \%$, minimum sample size was estimated to be 321 considering response rate to be $70 \%$. Convenience sampling was used to disseminate the questionnaires. Data was obtained from patients presenting to different departments in teaching hospitals after obtaining informed consent by two researchers. Confidentiality of participants was maintained by omission of questions from which they could be identified such as name and address while giving codes to the questionnaire booklets. Inclusion criteria entailed consenting adults (18 years and above) who could read or understand the questions. A self-administered structured questionnaire was developed to assess the knowledge and perception of patients regarding dental treatments, and 
hygiene practices while fasting. The questionnaire included items to record demographic variables of the participants. It also comprised of 7 items inquiring about influence of dental treatments such as filling and scaling etc. on fasting. Second part of questionnaire consisted of 3 items asking the participants whether hygiene measures such as brushing and use of mouthwash, miswaak affects fasting. The responses ranged from "maybe yes", "yes" to "maybe no", "no" and "don't know". These were then categorized respectively in to 'yes'; 'no' and 'don't know' for the purpose of analysis. Education level of the patients were dichotomized in to two groups; school graduates and college graduates. The high school diploma group includes participants who completed their primary and secondary education; graduates and postgraduates were categorized in college education. Incomplete questionnaires were discarded and not included in analysis. The data was entered and analyzed using SPSS statistical program (version 23). Results are reported as frequencies and percentages. Chi-squared test was used to observe differences between knowledge amongst genders, and education status of respondents. All $p$-values were two tailed and the significance was defined at $p<.05$

\section{Results}

A total of 380 responses were obtained out of 600 distributed, of which 6 were excluded due to being incomplete. Total sample included in the analysis was 374 . Summary of demographic variables are displayed in table 1. Majority of the participants were male, though much difference wasn't observed between genders as female participants formed almost half of the total count. Most (68.7\%) participants had completed their college degree followed by participants who were educated till primary or high school graduates (31.3\%). Majority of the participants were employed (55.9\%) with only $21.9 \%$ being unemployed.

Table 1: Demographic status

\begin{tabular}{ll}
\hline VARIABLE & \multicolumn{1}{c}{$\mathrm{N}(\%)$} \\
\hline Gender & \\
Male & $197(52.7 \%)$ \\
Female & $177(47.3 \%)$ \\
Age $^{1}$ & $29.4( \pm 11.5)$ \\
Education status & \\
Primary educated & $35(9.4 \%)$ \\
Secondary educated & $82(21.9 \%)$ \\
College educated & $257(68.7 \%)$ \\
Occupation & \\
Student & $83(22.2 \%)$ \\
Professional & $117(31.3 \%)$ \\
Businessmen & $30(8 \%)$ \\
Casual workers & $62(16.6 \%)$ \\
Unemployed & $82(21.9 \%)$ \\
\hline
\end{tabular}


${ }^{1}$ Displayed as Mean \pm S.D

[Insert Figure 1 here.]

Overwhelming majority of respondents thought induction of anesthesia (67.6\%) and procedure of extraction $(76.2 \%)$ broke fast. More than half $(52.9 \%)$ of the respondents assumed RCT to break the fast. Although majority perceived scaling to not break the fast, many $(45.5 \%)$ of respondent's views were in contrast.

Table 2 summarizes the responses of sample population according to education level. Amongst school graduates, majority perceived dental procedures to break fast whereas most college graduates thought otherwise. Procedures of scaling, impression taking, and crown cementation were not assumed to break the fast by both college and school graduates. According to majority of college educated respondents (77.8\%), filling was not considered to break fast whilst most (45.7\%) school graduates believed the procedure broke fast. Likewise, significant association was observed between education status of respondents and likelihood of perception of dental procedures breaking the fast except for procedure of scaling and extraction.

Table 2- Association of education and dental procedures likely to break the fast 


\section{Education}

Variable

$\mathrm{N}(\%)$

\section{Scaling}

College degree

$P$ value

Yes

$120(46.7 \%) \quad 50(42.7 \%)$

No

$132(51.4 \%)$

$61(52.1 \%)$

0.21

Don't know

$5(1.9 \%)$

$6(5.1 \%)$

\section{Filling}

\begin{tabular}{llll} 
Yes & $70(27.2 \%)$ & $59(50.4 \%)$ & \\
No & $178(69.3 \%)$ & $52(44.4 \%)$ & $<0.01$ \\
Don't know & $9(3.5 \%)$ & $6(5.1 \%)$ & \\
\hline Anesthesia & & & \\
Yes & $159(61.9 \%)$ & $94(80.3 \%)$ & \\
No & $85(33.1 \%)$ & $17(14.5 \%)$ & $<0.01$ \\
Don't know & $13(5.1 \%)$ & $6(5.1 \%)$ & \\
\hline RCT & & & \\
Yes & $129(50.2 \%)$ & $69(59 \%)$ & \\
No & $119(46.3 \%)$ & $27(23.1 \%)$ & \\
Don't Know & $9(3.5 \%)$ & $21(17.9 \%)$ & $<0.01$ \\
\hline Extraction & & & \\
Yes & $189(73.5 \%)$ & $96(82.1 \%)$ & \\
No & $60(23.3 \%)$ & $16(13.7 \%)$ & 0.09 \\
Don't know & $8(3.1 \%)$ & $5(4.3 \%)$ & \\
\hline Impression & & & \\
Yes & $81(31.5 \%)$ & $29(24.8 \%)$ & $<0.01$ \\
No & $165(64.2 \%)$ & $67(57.3 \%)$ & \\
Don't Know & $11(4.3 \%)$ & $21(17.9 \%)$ & \\
\hline Crown cuttng 8 Cem & &
\end{tabular}

\section{Crown cutting \& Cementation}

Yes

No

$58(22.6 \%)$

$34(29.1 \%)$

$<0.01$

Don't Know

$181(70.4 \%) \quad 59(50.4 \%)$


Overall, the male gender was most likely to consider that dental treatments broke the fast as compared to females. However, statistical significance was only observed between genders with respect to procedure of cavity filling $\left(\chi^{2}=9.74 ; p=<.01\right)$ and crown cementation $\left(\chi^{2}=8.64 ; p=.01\right)$.

The participants were also inquired about their views concerning the dental hygiene practices during fasting and if they thought those could potentially break their fast (figure 2).

[Insert Figure 2 here.]

As far as maintaining oral hygiene was concerned, majority respondents $(57.5 \%)$ of both educational background perceived brushing to break fast $\left(\chi^{2}=9.62 ; p=<.01\right)$. However, use of miswaak was not considered to break fast by most respondents in either group.

Significant difference was only observed amongst gender and use of miswaak $\left(\chi^{2}=8.21 ; p=.01\right)$; where most females responded negatively towards brushing and use of mouthwash as they assumed that these would break their fast. Conclusively, majority of respondents believed brushing teeth and using mouthwash to break fast. Overwhelming majority (88.2\%) did not associate use of miswaak with breaking of fast.

\section{Discussion}

This study aimed to evaluate the perception of dental patients concerning the influence of dental treatment on fasting. Most of respondents in the study thought that undergoing dental treatments did not invalidate fast though, oral hygiene practices broke fast. Procedures like scaling and filling were not thought to nullify fast by many. Since the use of rubber dam is common nowadays than it was previously, patients may be aware that it eliminates the risk of intake of any kind of liquid during filling and root canal treatments. In contrast, most of them thought that induction of anesthesia will invalidate the fast as, they may perceive introduction of anesthetic material in oral tissues to break fast. Also, it is generally assumed that any kind of inoculation breaks the fast. To tackle this sensitive issue, Islamic rulings were pronounced by scholars that administration of local anesthetics will not invalidate fast; only nutritional IV injections are not permitted during fasting. ${ }^{6}$ Although, it is doubtful if the patients are aware of any such ruling.

As far as maintaining oral hygiene is concerned, most respondents considered use of mouthwash, and tooth brushing to breakfast. This could be due to the presence of flavoring agents in toothpaste and mouthwash as consumption of flavorful components during fast is prohibited. However, using miswaak was not thought to be associated with breaking of fast. Miswaak is a small twig of a plant rubbed 
against the surfaces of teeth for the purpose of cleaning. The twig is reported to have some antimicrobial and plaque inhibitory effects. ${ }^{8}$ Conflicting opinions have been documented by different scholars where one school of thought suggests not brushing teeth during fasting state; though others have set a condition. It was proposed that tiny amount of toothpaste can be used to brush the teeth whilst fasting; ascertaining that the paste is not swallowed and spit out immediately.

Overall, females were more aware of impact of dental treatments and dental hygiene practices on fasting state as compared to males. While there were more males in the study, more females were educated till bachelor level. This could explain the more educated responses of females as compared to males as they were more educated and informed. Also, college educated respondents had relatively better awareness of procedures which did not break fast as compared to school graduated respondents. Similar results were observed in another study conducted in Malaysia where educated people were likely to be more knowledgeable about effect of dental treatments on fasting. ${ }^{9}$

This study holds importance especially amongst Muslim community since this domain hasn't been well researched. It can also be assumed that the results are generalizable to Muslim population anywhere as external validity was assured by devising an inclusion/exclusion criteria and collecting data at the same timing. In places where Muslims are a minority; this could prove to be helpful in understanding the knowledge and attitude of people who are fasting, as people often tend to miss appointments during Ramadan and even if they attend, usually do not undergo any form of treatment. ${ }^{7}$ The study also emphasizes on the importance of information regarding patient available to the dentist. For example, a study conducted amongst Muslims in India depicted that most of the respondents believed using eye drops during Ramadan would break their fast. ${ }^{10}$ On the contrary, no evidence exists that reinforces this notion; as the medicine is not taken orally. Moreover, the respondents indicated that they would not take the medicine in case of emergency as well. This implies that people who fast could be noncompliant with medications. ${ }^{10}$

It is important that dentists themselves should be updated with the religious affiliation of patients and the latest rulings in relation to dental treatments and medication. This is to avoid any inconvenience during treatment as patients may not visit their dentist even in emergency in fear of breaking their fast. ${ }^{11}$ Also, the dentists should ascertain that the patients are informed about the rulings such as the Islamic Fiqh Council and the Standing Committee for Academic Research and Issuing Fatwas, a Saudi Arabian organization that issues decrees in Islamic jurisprudence. ${ }^{12}$ One possible limitation that is associated with the study is that it could have benefited from including people living in rural areas since it is possible that their views may have differed.

\section{Conclusion}

The study indicated that the respondents in the sample were aware of most treatments permissible during fasting though, relatively uninformed of the influence of oral hygiene practices on fasting. Patient education should be focused on pre-Ramadan dental health evaluation and existing exemptions in 
Ramadan for people who require immediate treatment. Further research is recommended to determine the knowledge of non-Muslim practitioners with regards to treatments and practices which are allowed during fasting. This could help in formulating guidelines specific to those regions and health services authorities.

\section{Abbreviations}

OPD- outpatient department

RCT- root canal treatment

\section{Declarations}

\section{Ethics approval and consent to participate}

The study received ethical approval from the ethical review board (ERB) of University College of Dentistry, University of Lahore (ref no. UCD/ERCA/18/01a). Ethical approval was also sought from De'Montmorency college of dentistry. Written informed consent was obtained from the respondents and data was collected from those who consented to be a part of the study.

\section{Consent for publication}

Not Applicable

\section{Availability of data and materials}

The datasets used and/or analyzed during the current study are available from the corresponding author on reasonable request.

\section{Competing interests}

The authors declare that they have no competing interests.

\section{Funding}

The authors have no funds to declare.

\section{Authors' contributions}

KT was responsible for data analysis, interpretation and manuscript writing. 
$\mathrm{AH}$ was involved in study conception, data collection and data entry.

MH was involved in study conception and manuscript writing.

SD was involved in data analysis and manuscript writing.

ZA was involved in data entry and reporting.

MAB was involved in revision and approval of article.

All authors have read and approved the manuscript.

\section{Acknowledgements}

None

\section{References}

1. Abolaban H, Al-Moujahed A. Muslim Patients in Ramadan: A Review for Primary Care Physicians. Avicenna J Med. 2017; 7: 81-87.

2. Leiper JB, Molla A, Molla AM. Effects on Health of Fluid Restriction During Fasting in Ramadan. Eur J Clin Nutr. 2003;57 Suppl 2:30-38.

3. Jaleel MA, Raza SA, Fathima FN, Jaleel BNF. Ramadan and Diabetes: As-Saum (the Fasting). Indian J Endocrinol Metab. 2011; doi: 10.4103/2230-8210.85578

4. Ramadan J. Does Fasting During Ramadan Alter Body Composition, Blood Constituents and Physical Performance?. Med Princ and Pract. 2002;11 Suppl 2: 41-46.

5. Sakr AH. Fasting in Islam. J Am Diet Assoc. 1975;67:17-21.

6. Yusuf H. Ramadan: Six common misconceptions debunked'. BBC News. 2018. Available from: https://www.bbc.com/news/uk-44107850. (Accessed: 12 October 2019)

7. Peedikayil FC, Thomas A, Naushad M, Narayanan A. Management of Muslim Dental Patient While Fasting. Eur J Gen Dent. 2014; doi: 10.4103/2278-9626.126219

8. AlBarakati SF. Appointments Failure among Female Patients at a Dental School Clinic in Saudi Arabia. J Dent Educ. 2009;73:1118-1124.

9. Haque MM, Alsareii SA. A Review of the Therapeutic Effects of Using Miswak (Salvadora Persica) on Oral Health. Saudi Med J. 2015; doi: 10.15537/smj.2015.5.10785.

10. Mohammad FN, Said AH. Dental procedures during fasting: perceptions among muslims in malaysia. J Int Dent Med Res. 2018;12:597-601.

11. Kumar N, Jivan S. Ramadan and Eyedrops: The Muslim Perspective. Ophthalmology. 2007;114:2356-2360. 
12. Kochhar S, Singh K, Anandani C, Pani P, Bhullar KR, Bhullar A. Occurrence of Oral Health Beliefs and Misconceptions among Indian Population. J Dent Health Oral Disord Ther. 2014;1

13. Abolaban H, Al-Moujahed A. Muslim Patients in Ramadan: A Review for Primary Care Physicians. Avicenna J Med. 2017; 7: 81-87.

\section{Figures}

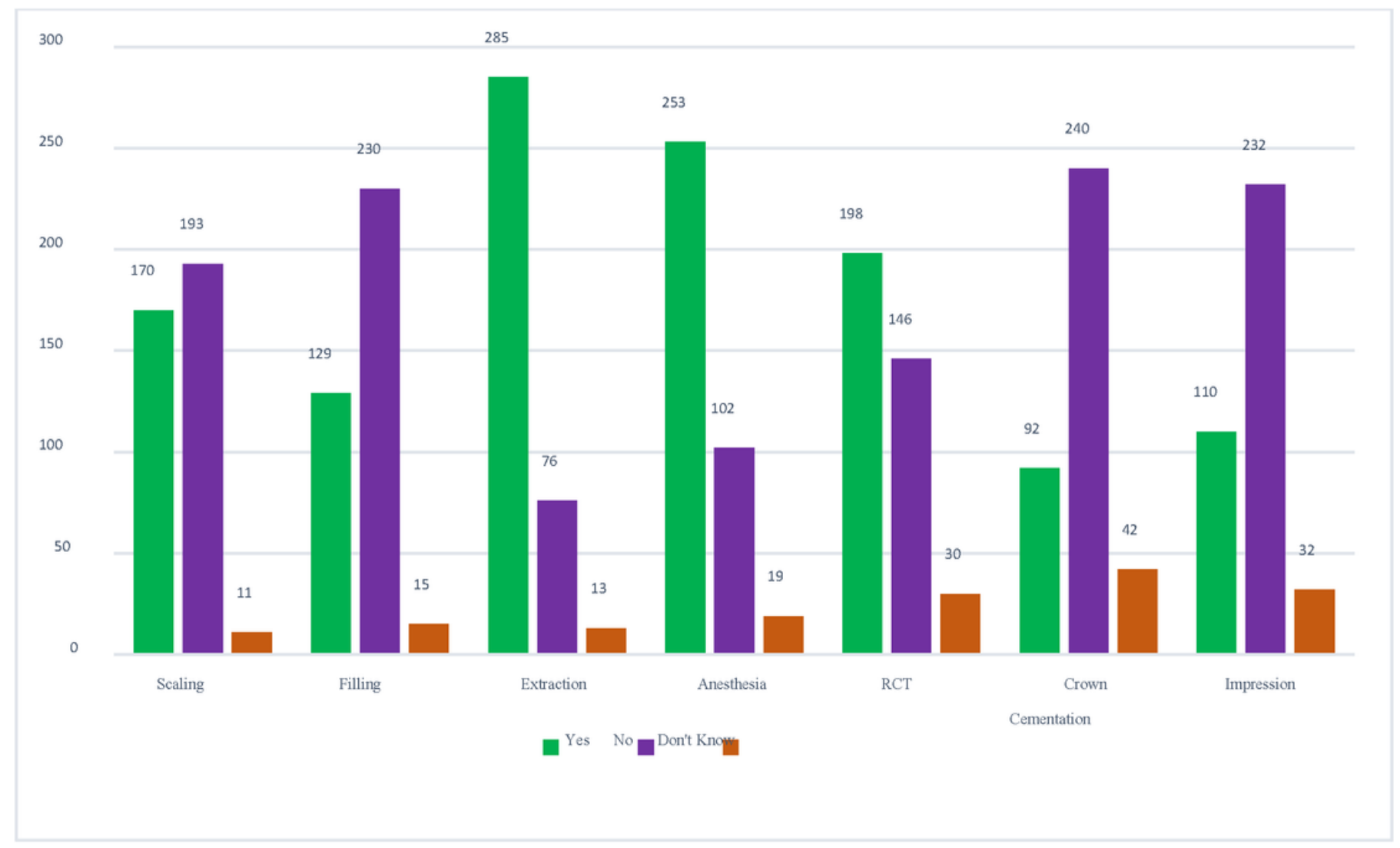

Figure 2

Respondents view of dental treatments permissible during fasting 


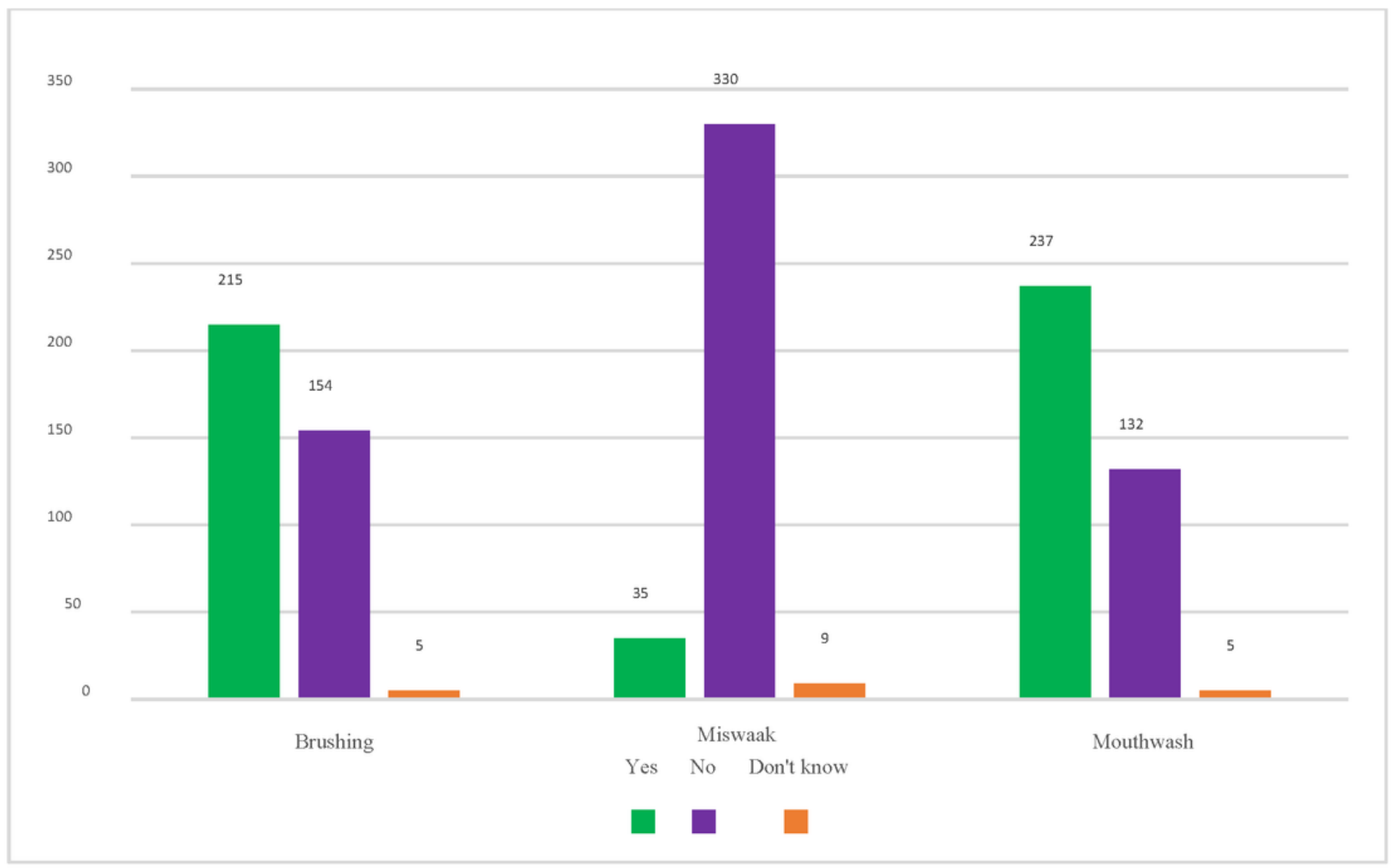

Figure 4

Perception of oral hygiene practices breaking fast 